\title{
The Role of Genetics in Sporadic GEP-NETs: A Comprehensive Review of the Literature
}

\author{
George Fotopoulos ${ }^{1,2}$, loannis Vathiotis ${ }^{1}$, George C. Nikou², \\ Konstantinos Syrigos ${ }^{1,3}$ \\ 'Oncology Unit, 3rd Department of Internal Medicine, Sotiria General \\ Hospital, National \& Kapodistrian University, Athens School of Medicine, \\ Athens, Greece \\ ${ }^{2}$ Multidisciplinary Unit of NET Management, 3rd Department of Internal \\ Medicine, Sotiria General Hospital, National \& Kapodistrian University, \\ Athens School of Medicine, Athens, Greece \\ ${ }^{3}$ Yale School of Medicine, New Haven, CT, USA;
}

Received 28 November 2016; Accepted 14 May 2017

\begin{abstract}
Neuroendocrine tumors (NETs) are composed of a heterogeneous group of malignancies from neuroendocrine cell compartments, with roles in both the endocrine and the nervous system. The majority of NETs are gastroenteropancreatic (GEP) in origin, arising in the foregut, midgut, or hindgut. The genomic landscape of GEP-NETs has been scarcely studied in terms of genomic profiling. The following algorithm was followed using the keywords neuroendocrine, genomics, targeted therapy, personalized medicine, gastroenteropancreatic and NET. The search was performed in PubMed and ScienceDirect database. Our current knowledge of sporadic GEP-NETs genetics must be further advanced to elucidate the molecular basis and pathogenesis of the disease, improve the accuracy of diagnosis, and guide tailor-made therapies.
\end{abstract}

Keywords: Neuroendocrine • Genomics • Targeted therapy • Personalized medicine • Gastroenteropancreatic • NET

(C) De Gruyter Open

\section{Introduction}

Neuroendocrine cell islets give rise to a diverse group of malignancies, thesocalledneuroendocrinetumors(NETs). In most cases, the NETs are gastroenteropancreatic (GEP) in origin, with their primary site located in the foregut, midgut or hindgut; both the nervous and the endocrine system are implicated in their formation [1]. Regardless of the perpetual increase in their reported incidence and prevalence based on the results of recent studies of the Surveillance, Epidemiology, and End Results (SEER) cancer registry and European studies, NETs remain very rare [2].

Although awareness of all the involved specialists such as gastroenterologists, endrocrinologists, medical oncologists and pathologists has resulted in higher rates of diagnosis, the therapeutic options and the outcome have not improved significantly. In patients with inoperable advanced disease and after the breakthrough use of somatostatin analogues the current therapeutic options also include, chemotherapy with agents such as doxorubicin, streptozocin, capecitabine, dacarbazine, temozolamide, fluouracil, oxaliplatin, the use of peptide receptor radionuclide therapy and more recently, the use of two molecularly targeted agents, everolimus and sunitinib [3].

Personalized medicine is defined as the use of an individual patient's molecular profile to inform diagnosis, prognosis, treatment, and prevention of cancer and has become a primary focus of many studies in oncology. The genomic landscape of GEP-NETs has been scarcely studied in terms of genomic profiling. The aim of this paper 
is to identify what is known about potent genomics and its products in terms of proteins, pathways and mutations in this setting and to explore the possible use of them in diagnosis, prognosis and management, if any apply.

Two debated terminologies have risen in the recent years, with the use of the term endocrine versus neuroendocrine and that of neoplasms instead of tumors. Also well differentiated gastrointestinal NETs are also termed carcinoid tumors. For the sake of uniformity in this paper the term NETs will be used

\section{Material and Methods}

A literature search was performed with end-date June 2016 and a year span of the last ten years. The following algorithm was followed using the keywords neuroendocrine, NENs genomics, targeted therapy, personalized medicine, gastroenteropancreatic. Once duplicates were identified and removed, the retrieved articles were then reviewed by two separate authors for inclusion or exclusion. Case reports, non English articles, and articles that their context could not be accessed and also articles dealing with GEP-NETs in the setting of a familial syndrome were excluded. Furthermore the reference list of the included articles was further explored for potentially relevant studies. The search was performed in PubMed and ScienceDirect database.

\section{Genetics in GEP-NETs}

One could identify two sets of genes involved in GEPNETs, the one set found in pancreatic NETs (pNETs) and the other set in the rest of NETs also (Table 1).

The genes and its products involved in pNETs are ABTB1, ZNF322A, CD36, TP53, DAXX, ARTX, TSC1-2, $Y Y 1, F E V, A D C Y 2$ and NR4A2 [4,5].

ABTB1 gene incorporates an ankyrin repeat and two BTB/POZ domains. The gene's product, expressed in fetal rather than adult tissues, is considered to be entangled in normal development, enhancing protein interconnections. Expression of this gene is activated by the phosphatase and tensin homolog, a tumor suppressor [6]. Alterations in the form of gains affecting the $6 p 22.2-22.1$ region where this gene lies have been found in meningiomas [7]. Its importance in NETs and tumorigenesis is not well established but one could argue that the interaction with the homolog acting as tumor suppression is altered thus leading to tumorigenesis.

In the same region lies the ZNF322A gene encoding a classical Cys2His2 zinc finger transcription factor. Although it has been associated with lung cancer
Table 1. Genes and products involved in tumorigenesis of NETs.

\begin{tabular}{c|c}
\hline GEP NETs & NETs \\
\hline \hline ABTB1 & SMARCCB1 \\
ZNF322A & STK11 \\
CD36 & RET \\
TP53 & BRAF \\
DAXX & CCNE1 \\
ARTX & \\
TSC1-2 & \\
YY1 & \\
FEV & \\
ADCY2 & \\
NR4A2 & \\
\hline
\end{tabular}

formation, evidence remains controversial. ZNF322A gene upregulation boosted cell proliferation, migration and invasion. Furthermore, its knockdown diminished cell growth, invasion and also metastasis both in vitro and in vivo. Alpha-adducin (ADD1), cyclin D1 (CCND1), and p53 have been identified as potential downstream ZNF322A targets by quantative proteomic analysis [8].

CD36 is a product of a gene located in chromosome 7. Gains and amplifications on the short arm of it have previously been detected in many malignant tumors such as sarcomas and carcinomas [9, 10]. This product is an eminent adhesion molecule and hepatocyte growth factor, as well as a regulatory protein involved in cell growth, proliferation, motility, matrix invasion and angiogenesis. As a result, CD36 constitutes a core element in tumorigenesis and dissemination. It also exhibits a potential substrate-dependent antiangiogenic potential [11]. Such alterations have been found in various NETs specimens so it seems that there is some implication of this gene in the pathogenesis.

TP53 gene, situated in the short arm of chromosome 17 , represents the most frequently mutated gene $(>50 \%)$ in malignant tumors, indicating its pivotal role in carcinogenesis. TP53 gene encodes proteins that bind to DNA and adjust gene expression to prevent mutations of the genome [12]. If the TP53 gene is damaged, tumor suppression is critically exposed. Mutagens (chemicals, radiation, or viruses) can also hamper its function, increasing the likelihood for uncontrolled cell division. Deletions and/or mutations affecting the 17p13.1 locus where TP53 lies have been found in atypical lung carcinoids and other NET tumors [13]. It comes as no surprise therefore that such a gene implicated in almost every aspect of tumorigenesis in various malignancies seems to have a role in NETs. 
It has been suggested that ATRX and DAXX mutations are not involved in the initial phase of tumorigenesis but instead represent late events in the malignant cascade [14]. The proposed pathogenic mechanism of ATRX and DAXX deficiency is the induction of the alternative lengthening of telomeres (ALT) phenotype, in pNETs among other solid tumors. ALT phenotype triggers genome rearrangements, defects in the G2/M checkpoint control, prominent micronucleation and altered doublestrand break repair mechanisms [15]. ATRX and DAXX mutated sporadic pNETs feature chromosomal instability and are associated with worse prognosis, in terms of advanced stage, metastatic disease, reduced tumorfree intervals and decreased survival [14].

Insulin producing pancreatic NETs, such as insulinomas, exhibiting signs and symptoms of hypoglycemia, have been related to recurrent somatic mutations in YY1 gene. The Thr372Arg recurrent mutation in the previously mentioned gene has been shown to result in neomorphic effects and altered transcription. High levels of genomic instability are associated with insulinomas with more prominent malignant features [16].

TSC1 and TSC2 form a tumor suppressor complex that acts as a negative regulator of the mammalian target of rapamycin (mTOR) signaling complex by maintaining direct control of the small GTPase Rheb via the GTPase activating protein (GAP) domain of TSC2 [17]. The importance of this pathway in carcinogenesis is well established, but what is of greater importance is that there are inhibitors available to target this pathway.

FEV gene belongs to the superfamily of transcription factors ETS. The ETS family is present throughout the body and is involved in a wide variety of functions including the regulation of cellular differentiation, cell cycle control, cell migration, cell proliferation, apoptosis and angiogenesis. Its factors act as transcriptional repressors, transcriptional activators, or both [18]. FEV is highly upregulated in malignant pancreatic NETs.

ADCY2 gene encodes an enzyme typically expressed in the human brain. It is a member of adenylyl cyclase family. This membrane-associated enzyme catalyzes the production of secondary messenger cyclic adenosine monophosphate (CAMP) from ATP. ADCY2 has also been shown to precipitate phosphor-acidification, along with glycogen synthesis and breakdown [19]. This is a gene also amplified in pNETs especially those of higher grade.

NURR1 protein, produced by NR4A2 geneexpression, represents a member of the nuclear receptor family of intracellular transcription factors, with four distinct isoforms. Additional alternate splice variants may exist, but their full-length nature has not been determined [20].
Although its role has not been fully defined yet, NR4A2 gene is highly upregulated in pNETs.

In the rest of NETs, genes that are involved in tumorigenesis are SMARCCB1, STK11, RET, BRAF and CCNE1.

SMARCCB1 is a gene with two transcript variants encoding different isoforms. Its protein product serves as a tumor suppressor, being part of a cluster that relieves repressive chromatin structures, in order to expedite the transcriptional machinery's access to its targets. Mutations in the above gene have been linked to malignant rhabdoid tumors [21], as well as grade II/ III NETs; SMARCCB1 mutations can be targeted with pazopanib [22].

STK11, still another tumor suppressor gene, encodes a member of the serine/threonine kinase family, regulating cell polarity. In addition, recent studies have spotted a large number of somatic mutations of the LKB1 gene present in large number of solid tumors, including cancers of the cervix, breast, intestine, testicle, pancreas and skin and also metastatic GEPNETs of higher grade [23]. Again of great importance is the targeting of this pathway with everolimus, another option in our treatment strategy [22].

On the contrary, RET proto-oncogene product is a receptor tyrosine kinase for members of the glial cell line-derived neurotrophic factor (GDNF) family of extracellular signaling molecules. Chromosomal rearrangements create a hybrid oncogene, leading to the production of a fusion protein that combines the $\mathrm{C}$-terminal region of the RET protein with the $\mathrm{N}$-terminal region of another protein. The result is uncontrolled activation of the RET kinase. These types of mutations are primarily associated with papillary thyroid carcinoma, and the fusion oncoproteins generated are termed RET/PTC proteins [24]. Mutations in RET gene have been implicated in the development of sporadic GEPNETs but mainly in the context of the hereditary cancer syndrome known as multiple endocrine neoplasia type 2 (MEN2) [25].

In a study by Park et al. [26] BRAF V600E (G1 NET from rectum and two G3 NETs from colon) and BRAF G593S (G2 NET from pancreas) missense mutations $(9.1 \%)$ in an independent cohort of 44 GEP-NETs from the rectum $(n=26)$, colon $(n=7)$, pancreas $(n=4)$, small intestine $(n=3)$, stomach $(n=3)$ and appendix $(n=1)$ were discovered. All tumor specimens were obtained before chemotherapy. They concluded that BRAF V600E mutation is likely to result in resistance to pazopanib but acts as an actionable mutation in metastatic GEP-NETs patients.

CCNE1 is a gene encoding the G1/S-specific cyclin-E1 protein. The above cyclin forms a cluster 
with CDK2, functioning as its regulatory subunit. Accumulating at the G1-S phase boundary and degraded through $S$ phase, cyclin-E1's activity is requested for cell cycle G1/S transition. Upregulation of this gene has been detected in solid tumors, generating chromosome instability, thus further contributing to malignant transformation [27]. In a series of 14 metastatic GEP-NETs in one case amplification of this gene was observed [4]. What is more important however is the potent targeting of it with cyclin dependent kinase (CDK) inhibitors [22].

\section{Discussion}

To this day, treatment options for GEP-NETs are somewhat limited with regard to other malignancies. Somatostatin analogues, chemotherapy, peptide receptor radionuclide therapy and sunitinib and everolimus are the main agents we have at our disposal. Still, many questions remain unanswered such as which is the best sequencing in these agents, which is the optimal chemotherapeutic agent and how can we combine these agents.

\section{References}

[1] Williams ED, Sandler M. The classification of carcinoid tumours. Lancet. 1963;1: 238-239.

[2] Yao JC, Hassan M, Phan A et al. One hundred years after «carcinoid»: epidemiology of and prognostic factors for neuroendocrine tumors in 35,825cases in the United States. J Clin Oncol. 2008; 26: 30633072

[3] https://www.nccn.org/professionals/physician_gls/ pdf/neuroendocrine.pdf

[4] Niklas Gebauer, Christian Schmidt-Werthern, Veronica Bernard et al.Genomic landscape of pancreatic neuroendocrine tumors. World J Gastroenterol 2014 December 14; 20(46): 1749817506

[5] Joakim Crona , Britt Skogseid. Genetics of neuroendocrine tumors. European Journal of Endocrinology(2016) 174, R275-R290

[6] Ken-Shwo Dai, Wei Wei, Choong-Chin Liew. Molecular Cloning and Characterization of a Novel Human Gene Containing Ankyrin Repeat and Double BTB/POZ Domain. Biochemical and Biophysical Research Communications 273, 991996 (2000)

[7] Pérez-Magán E, Rodríguez de Lope A, Ribalta T et al. Differential expression profiling analyses
Nowdays there is a bigger awareness for this disease and therefore the incidence of NETs is rising, especially in the light of better diagnostic techniques and more trained pathologists. All these unmet needs must be dealt with and explored so we could have the best treatment available for these patients. But as we are entering the era of genomic profiling and personalized medicine steps must be taken also at this direction.

In contrast to other malignancies, for the diagnosis of NET a tissue sample is mandatory. This is fortunate because there is abundance of sample for molecular profiling and genetic testing. As we mentioned earlier, mutations have been discovered in this setting where we can target them such as BRAF, CCNE1, SMARCCB1 and STK11. All these need to be verified in larger cohorts and also to look for more mutations we can target upon such as EGFR, ALK, KRAS, cKIT known for their oncogenic drive in other tumors. Furthermore we need to design clinical trials addressing such issues and mainly to answer specific questions, such as which are the best biomarkers, if any, in GEP-NETs.

The time has come now more than ever to apply what we learn from translational research to clinical trials and hopefully one day to clinical practice.

identifies downregulation of $1 p, 6 q$, and $14 q$ genes and overexpression of $6 p$ histone cluster 1 genes as markers of recurrence in meningiomas. Neuro Oncol 2010; 12: 1278-1290

[8] Jen J1, Lin LL2, Chen HT3 et al. Oncoprotein ZNF322A transcriptionally deregulates alphaadducin, cyclin D1 and p53 to promote tumor growth and metastasis in lung cancer. Oncogene,2016 May 5;35(18):2357-69

[9] Aragane H, Sakakura C, Nakanishi M et al. Chromosomal aberrations in colorectal cancersand liver metastases analyzed by comparative genomic hybridization. Int J Cancer 2001; 94: 623-629

[10] Schmidt H, Taubert H, Würl P et al. Gains of $12 q$ are the most frequent genomic imbalances in adult fibrosarcoma and are correlated with a poor outcome. Genes Chromosomes Cancer 2002; 34: 69-77

[11] Hale JS, Li M, Sinyuk M, Jahnen-Dechent W et al. Context dependent role of the CD36-thrombospondin-- histidine-rich glycoprotein axis in tumor angiogenesisand growth. PLoS One 2012; 7: e40033

[12] May P, May E. Twenty years of p53 research: structural and functional aspects of the p53 protein. Oncogene 1999, 18 (53): 7621-36 
[13] Vousden $\mathrm{KH}$, Prives C. Blinded by the Light: The Growing Complexity of p53. Cell 2009; 137: 413431

[14] Marinoni I, Kurrer AS, Vassella E et al. Loss of DAXX and ATRX are associated with chromosome instability and reduced survival of patients with pancreatic neuroendocrine tumors. Gastroenterology 2014 ,146, 453-460.

[15] Lovejoy CA, Li W, Reisenweber S et al. Loss of ATRX, genome instability, and an altered DNA damage response are hallmarks of the alternative lengthening of telomeres pathway. PLoS Genetics 20128.

[16] Jonkers YM, Claessen SM, Perren A et al. DNA copy number status is a powerful predictor of poor survival in endocrine pancreatic tumor patients. Endocrine-Related Cancer 200714 769-779.

[17] Guertin DA, Sabatini DM . Defining the role of mTOR in cancer. Cancer Cell 2009, 12 (1): 9-22.

[18] Verger A, Duterque-Coquillaud M. When Ets transcription factors meet their partners. Bioessays. 2002 Apr;24(4):362-70.

[19] Li YX, Jin HG, Yan CG et al. Molecular cloning, sequence identification, and gene expression analysis of bovine ADCY2 gene. Mol Biol Rep. 2014 Jun;41(6):3561-8

[20] Sacchetti P, Carpentier R, Ségard P et al. Multiple signaling pathways regulate the transcriptional activity of the orphan nuclear receptor NURR1. Nucleic Acids Res. 2006;34(19):5515-27
[21] Lee D, Sohn H, Kalpana GV et al. Interaction of E1 and hSNF5 proteins stimulates replication of human papillomavirus DNA. Nature 399 (6735): 487-9

[22] Le Tourneau C, Paoletti X, Servant N, et al. Randomised proof-of-conceptphase II trial comparing targeted therapy based on tumour molecularprofiling vs conventional therapy in patients with refractory cancer: results ofthe feasibility part of the SHIVA trial. Br J Cancer. 2014; 111: 17-24.

[23] Sanchez-Cespedes M. A role for LKB1 gene in human cancer beyond the Peutz-Jeghers syndrome. Oncogene 200726 (57): 7825-32.

[24] Knowles PP, Murray-Rust J, Kjaer S et al. Structure and chemical inhibition of the RET tyrosine kinase domain. J Biol Chem. 2006 Nov 3;281(44):3357787

[25] Xiao-Ping Qi,1, ${ }^{*}$ Ju-Ming Ma,1 Zhen-Fang Du et al. RET Germline Mutations Identified by Exome Sequencing in a Chinese Multiple Endocrine Neoplasia Type 2A/Familial Medullary Thyroid Carcinoma Family. PLoS One. 2011; 6(5): e20353.

[26] Park C, Ha SY, Kim ST et al. Identification of the BRAF V600E mutation in gastroenteropancreatic neuroendocrine tumors. Oncotarget. 2016 Jan 26;7(4):4024-35

[27] Lew DJ, Dulić V, Reed SI. Isolation of three novel human cyclins by rescue of $\mathrm{G} 1$ cyclin (CIn) function in yeast. Cell. 1991 Sep 20;66(6):1197-206. 